\title{
Review: various interventions increase smoking cessation rates
}

\author{
Lancaster T, Stead L, Silagy C, et al, for the Cochrane Tobacco Addiction Review Group. Effectiveness of interventions to help \\ people stop smoking: findings from the Cochrane Library. BMJ 2000 Aug 5;321:355-8.
}

\section{QUESTION: Are smoking cessation interventions effective?}

\section{Data sources}

Reviews were identified by searching the Cochrane Library.

\section{Study selection}

Reviews were selected if they included randomised controlled trials of interventions to reduce or prevent tobacco use that had $\geqslant 6$ months of follow up with outcomes of sustained abstinence or point prevalence stop rates.

\section{Data extraction}

Extracted data included interventions and outcomes.

\section{Main results}

20 systematic reviews were available in the Cochrane Library. One review (including 31 trials and $>26000$ participants who were smokers) examined simple advice given by doctors during routine care and showed that the intervention increased quit rates (weighted odds ratio [OR] $1.7,95 \%$ CI 1.5 to 2.0 ). Another review of individual counselling given by nurses also showed increased stopping rates.

Behavioural interventions for smoking cessation, in the forms of individual counselling (1 review) or group therapy (1 review), showed increased stopping rates. The review of individual counselling (9 trials) showed that it was more effective than brief advice or usual care (weighted OR 1.6, CI 1.3 to 1.9). Group therapy was more effective than self help materials but was not more effective than interventions involving personal contact. 1 review of aversion therapy (24 trials), in which the pleasurable stimulus of smoking was paired with an unpleasant stimulus, found little evidence of beneficial effect. 1 review showed that self help interventions had no additional benefit over brief personal advice but were more effective than no intervention (weighted OR 1.2, CI 1.0 to 1.5). A comparison of self help interventions ( 8 trials) showed that personalised materials were more effective than standard materials (weighted OR 1.4, CI 1.1 to 1.8) and that some benefit could also be provided by telephone support (7 trials).

A review of nicotine replacement treatment $(>90$ trials) showed that all forms of this treatment were effective (weighted OR 1.7, CI 1.6 to 1.8). A review of anxiolytic and antidepressant agents showed that anxiolytics were not effective but that the antidepressants bupropion and nortriptyline increased stopping rates. A review of the antihypertensive clonidine (6 trials), showed effectiveness (weighted OR 1.9, CI 1.3 to 2.7), but its usefulness was limited by its side effect profile. A review of lobeline showed no consistent effect of treatment at 6 weeks. Reviews of acupuncture, hypnotherapy, and exercise showed limited evidence of effectiveness for smoking cessation.

\section{Conclusion}

Counselling by doctors and nurses, behavioural interventions (individual counselling or group therapy), nicotine replacement treatment, and several pharmacological interventions (such as the antidepressants bupropion and nortriptyline) increase smoking cessation rates.

\section{COMMENTARY}

Lancaster et al have done a credible overview of smoking cessation interventions, making this valuable information in the Cochrane Library accessible to a broader audience. The authors have summarised the results of 20 relevant, methodologically rigorous, systematic reviews from the Cochrane Database of Systematic Reviews. An abstract of each review can be accessed free of charge (http:// www.update-software.com).

The findings of this review will not be news to many people who are generally familiar with research on smoking cessation. For the most part, the conclusions mirror the recommendations of the recently updated US Public Health Service's (USPHS) Clinical Practice Guideline on Treating Tobacco Use and Dependence. ${ }^{1}$ It is reassuring that despite the use of somewhat different meta-analytic and interpretive methods, both reports agree that all forms of nicotine replacement appear to be about equally effective, sustained release bupropion is efficacious, all forms of counselling are effective, self help materials are of limited effectiveness, and anxiolytic agents are ineffective. The USPHS report is more supportive of aversive smoking techniques than is the Cochrane review. The Cochrane review interprets the evidence as favouring individually tailored self help material over standard ones, whereas the USPHS report calls for further study. The USPHS interprets the evidence on combining 2 forms of nicotine replacement treatment favourably and recommends this approach, whereas the Cochrane review is more cautious and calls for further study. Both the USPHS report and the 20 individual Cochrane reviews are much richer in detail than is this overview and are highly recommended reading for those with a serious interest in smoking cessation.

Roger Luckmann, MD, MPH University of Massachusetts Medical School Worcester, Massachusetts, USA

1 US Public Health Service. Treating tobacco use and dependence: summary. Rockville, MD: U.S. Public Health Service, June 2000. on http://www.surgeongeneral.gov/tobacco/.
Sources of funding: $U K$ National Health Service Research and Development

Programme and Imperial Cancer Research Fund.

For correspondence: Dr T Lancaster, Imperial Cancer Research Fund General Practice Research Group, Department of Primary Health Care, University of Oxford, Institute of Health Sciences, Oxford OX3 7LF UK. Fax $+44(0) 1865227137$.

Abstract and commentary also appear in

Evidence-Based Mental Health. A modified version of this abstract appears in Evidence-Based Nursing. 